\title{
Molecular analysis of Streptococcus pneumonia strains involved in vaccine failure in the Czech Republic
}

\section{National Institute of Public Health, Centre for Epidemiology and Microbiology, Prague, Czech Republic \\ J. Kozakova, Z. Okonji jana.kozakova@szu.cz}

Material and methods

Background

S. pneumoniae strains are referred to the National Reference Laboratory for Streptococcal Infections from all over the Czech Republic.

In accordance with the international definition, the pneumococcal vaccine failure was defined as IPD caused by a PCV serotype of S. pneumoniae in a child who meets at least one of the following criteria:

- aged $<12$ months when presenting with the onset of IPD within no more than 14 days after receiving PCV dose 2;

- aged $\geq 12$ months when presenting with the onset of IPD after receiving one dose of PCV (regardless of the number of previous doses).

The identification of $S$. pneumoniae was based on the optochin test on Columbia blood agar with a bile solubility test or real-time PCR.

The identification of S. pneumoniae was based on optochin test on Columbia blood agar with a bile solubility test or real-time PCR. The classification of $S$. pneumoniae into serotypes or serogroups is routinely done by the NRL for Streptococcal Infections using the Quellung reaction in combination with end-point multiplex PCR (mPCR). To characterise S. pneumoniae in more detail, multilocus sequence typing (MLST) is used. MLST provides data on allelic variants of the $S$. pneumoniae housekeeping genes $\operatorname{aroE}, g d h, g k i$ recP, spi, xpt, and $d d l$, on which basis sequence types (ST) and possibly also clonal complexes (cc) are determined. To assess MLST data, the DNASTAR Lasergene 12 Core Suite software was used. Dendrograms were generated from MLST data from the PubMLST database using the SplitsTree 4 (version 4.14.5) and Inscape 0.92 software packages.

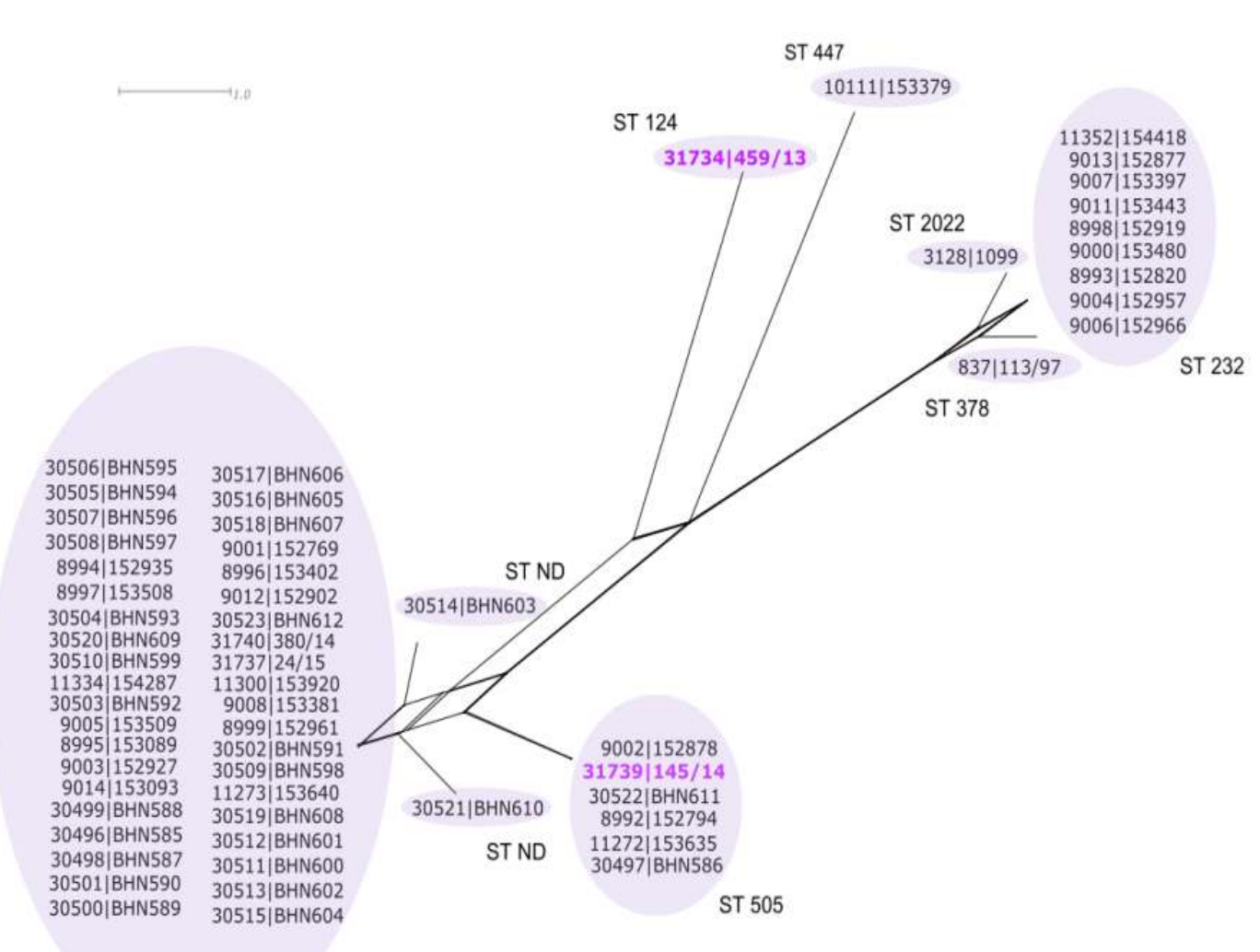

First conjugate pneumococcal vaccine registered in the Czech Republic was PCV7 in 2005. In following years PCV10 and PCV13 were registered and infants with underlying diseases were vacein infants in 2010 in the scheme $3+1$

The present study focuses on molecular characterisation of Streptococcus pneumoniae isolates from patients previously immunised with pneumococcal conjugate vaccine whose IPD was caused by a vaccine serotype in 2012 - 2014. The target group were children under 5 years of age. Results

Cases of IPD are recorded in the Czech Republic across age groups, including the smallest children, every year. In total, 335 cases of IPD were entered in the surveillance database in the Czech Republic in 2012, of which 15 in children under 5 years of age. In 2013, 28 of 424 cases of IPD occurred in children under 5 years of age. In 2014, 25 of 337 cases of IPD were reported in children under 5 years of age.

In 2012 to 2014, eight cases of vaccine failure were observed in children under 5 years of age. The eight isolates of S. pneumoniae from 2012 ( $n=1$ ), 2013 $(n=4)$, and $2014(n=3)$ were analysed by molecular methods Table 1.

Based on the serotyping results, the isolates of S. pneumoniae were assigned to three serotypes: $1(n=4), 14(n=2)$, and $3(n=2)$. Serotype 3 has long been the most common cause of IPD in the Czech Republic, and serotypes 14 and 1 are also involved in many cases every year. They are also detected in healthy carriers in the Czech Republic.

MLST classified all four isolates of serotype 1 into ST306, which is the dominant sequence type among the isolates of serotype 1 both in the Czech Republic and worldwide. Both isolates of serotype 14 were assigned to ST124, the sequence type which is very common among serotype 14 isolates in the Czech Republic as well as in other countries such as England. However, serotype 14 is diverse, and the distribution of its STs varies. Variability was also observed in serotype 3, with one isolate belonging to ST505 and the other to ST124. ST505 is common among serotype 3 isolates although sequence types ST180, ST260, or ST232 are more frequent. Of highest interest is the isolate of serotype 3 and ST124, as such a combination of serotype 3 and sequence type ST124 has not yet been observed. It is the first case to be reported to the PubMLST database in the world. The incidence and relatedness of STs in serotype $3 \mathrm{~S}$. pneumoniae reported from the Czech Republic to the international PubMLST database is illustrated by the dendrogram Figure 1. It shows a relatively high heterogeneity of isolates, with ST232 and ST505 being more common and ST180 being dominant. The two isolates recovered from cases of vaccine failure in children under 5 years of age are highlighted in colour.

Table 1 Strains of Streptococcus pneumoniae, pneumococcal vaccine failure in children under 5 years of age, Czech Republic, 2012-2014

\begin{tabular}{|c|c|c|c|c|c|c|c|c|c|c|c|c|c|c|c|c|}
\hline \multirow[b]{2}{*}{$\begin{array}{c}\text { Isolate } \\
\text { No. }\end{array}$} & \multirow[b]{2}{*}{ Serotype } & \multirow[b]{2}{*}{$\begin{array}{c}\text { Vaccine type } \\
\text { /vaccination } \\
\text { scheme }\end{array}$} & \multirow[b]{2}{*}{$\begin{array}{l}\text { Date of the last } \\
\text { dose / dose No. }\end{array}$} & \multirow[b]{2}{*}{$\begin{array}{c}\text { Child's } \\
\text { age }\end{array}$} & \multirow{2}{*}{$\begin{array}{l}\text { Date of } \\
\text { clinical } \\
\text { specimen } \\
\text { collection }\end{array}$} & \multirow[b]{2}{*}{$\begin{array}{c}\text { Clinical } \\
\text { specimen }\end{array}$} & \multirow[b]{2}{*}{$\begin{array}{c}\text { Primary } \\
\text { diagnosis }\end{array}$} & \multicolumn{9}{|c|}{ MLST } \\
\hline & & & & & & & & aroE & $g d h$ & gki & recP & spi & $x p t$ & $d d l$ & $\begin{array}{c}\text { Sequence } \\
\text { type } \\
\text { (ST) }\end{array}$ & $\begin{array}{l}\text { Clonal } \\
\text { complex } \\
\text { (cc) }\end{array}$ \\
\hline $311 / 12$ & 14 & PCV10 / 3+0 & 20.2.2012 / 3 dose & $9 \mathrm{~m}$ & 18.4.2012 & blood & pneumonia & 7 & 5 & 1 & 8 & 14 & 11 & 14 & 124 & \\
\hline $117 / 13$ & 14 & PCV10 / 2+1 & 20.4.2010/3 dose & $4 y$ & 11.2 .2013 & blood & pneumonia & 7 & 5 & 1 & 8 & 14 & 11 & 14 & 124 & \\
\hline $459 / 13$ & 3 & PCV13 / 3+0 & 26.7.2012 / 3 dose & $1 \mathrm{y}$ & 10.7.2013 & blood & pneumonia & 7 & 5 & 1 & 8 & 14 & 11 & 14 & 124 & \\
\hline $522 / 13$ & 1 & PCV10 / 3+1 & 3.1.2010 / 4 dose & $4 y$ & 20.9 .2013 & blood & pneumonia & 12 & 8 & 13 & 5 & 16 & 4 & 20 & 306 & 18 \\
\hline $584 / 13$ & 1 & PCV10 / 3+1 & 11.11.2010 / 4 dose & $3 y$ & 12.10 .2013 & blood & meningitis & 12 & 8 & 13 & 5 & 16 & 4 & 20 & 306 & 18 \\
\hline $145 / 14$ & 3 & PCV13 / 3+1 & 15.8.2013 / 4 dose & $1 \mathrm{y}$ & 7.2.2014 & blood & pneumonia & 46 & 8 & 2 & 10 & 6 & 1 & 22 & 505 & \\
\hline $465 / 14$ & 1 & PCV13 / 3+1 & 15.12.2011 / 4 dose & $3 y$ & 10.6.2014 & blood & pneumonia & 12 & 8 & 13 & 5 & 16 & 4 & 20 & 306 & 18 \\
\hline $551 / 14$ & 1 & PCV10 / 3+1 & 9.8.2011 / 4 dose & $4 y$ & 29.7.2014 & blood & sepsis & 12 & 8 & 13 & 5 & 16 & 4 & 20 & 306 & 18 \\
\hline
\end{tabular}

\section{Conclusion}

Results of a pilot project in the Czech Republic are presented. The monitoring and further characterisation of cases of vaccine failure should be continued. The data obtained are of high relevance to the surveillance of invasive pneumococcal disease in the Czech Republic.

\section{Acknowledgement}

Supported by grant no.17-29256A from the Ministry of Health of the Czech Republic. All rights reserved 